\title{
A Comparison of Tournaments and Contracts
}

\section{Citation}

Green, Jerry R. and Nancy L. Stokey. 1983. A comparison of tournaments and contracts. Journal of Political Economy 91(3): 349-364.

\section{Published Version}

http://dx.doi.org/10.1086/261153

\section{Permanent link}

http://nrs.harvard.edu/urn-3:HUL.InstRepos:3203644

\section{Terms of Use}

This article was downloaded from Harvard University's DASH repository, and is made available under the terms and conditions applicable to Other Posted Material, as set forth at http:// nrs.harvard.edu/urn-3:HUL.InstRepos:dash.current.terms-of-use\#LAA

\section{Share Your Story}

The Harvard community has made this article openly available.

Please share how this access benefits you. Submit a story.

Accessibility 


\section{A Comparison of Tournaments and Contracts}

\section{Jerry R. Green}

Harvard University

\section{Nancy L. Stokey}

Northwestern University

Tournaments, reward structures based on rank order, are compared with individual contracts in a model with one riskneutral principal and many risk-averse agents. Each agent's output is a stochastic function of his effort level plus an additive shock term that is common to all the agents. The principal observes only the output levels of the agents. It is shown that, in the absence of a common shock, using optimal independent contracts dominates using the optimal tournament. Conversely, if the distribution of the common shock is sufficiently diffuse, using the optimal tournament dominates using optimal independent contracts. Finally, it is shown that for a sufficiently large number of agents, a principal who cannot observe the common shock but uses the optimal tournament does as well as one who can observe the shock and uses independent contracts.

\section{Introduction}

At the Olympics prizes are awarded, not on the basis of absolute performance, but on the basis of relative performance. Similarly, in most organizations one of the most important rewards is promotion. If the hierarchical structure of the organization is fixed, employees at

We wish to thank Larry Jones and Paul Milgrom for very helpful comments. This work was supported by NSF grant APR77-06999 to Harvard University and by an IBM Research Professorship and the Center for Advanced Studies in Managerial Economics and Decision Sciences at Northwestern University. 
any one echelon are competing for a fixed, smaller number of positions at the next higher echelon. The goal for these employees is not just to do well but to do better than their peers.

The existing literature on incentives and contract design has been concerned primarily with the case where a principal employs only one agent and rewards him on the basis of absolute performance. Exceptions include several recent papers about tournaments-reward structures based on rank order-and more general compensation schemes for multi-agent settings. ${ }^{1}$

Most closely related to our work is the paper by Lazear and Rosen (1981). They examine, among other questions, the problem of a riskneutral principal with two agents, where the output of each agent depends stochastically on his own effort and on an additive shock that is common to both. The agents do not know the value of the common shock at the time they choose their effort levels; they do know its distribution. Lazear and Rosen compare three compensation schemes: a linear piece rate, comparison against a fixed standard, and a tournament. In the first, each agent's compensation is a linear function of his output; in the second, each agent receives one of two fixed payments, depending on whether his output is above or below a specified standard; and in the third, the agents compete against each other for two fixed prizes, allocated on the basis of the rank order of their output levels.

Lazear and Rosen show that if the agents are risk neutral, under any of the above schemes the moral hazard problem can be costlessly avoided by shifting all risk onto the agents. They also show through a series of examples that if the agents are risk averse, the relative ranking of the three schemes depends upon the relative variances of the common shock and the stochastic component of output attributable to effort. If the variance of the common shock is relatively large, a tournament will outperform the other two schemes; if the variance of the (stochastic) component of output attributable to effort is relatively large, one of the other two schemes will dominate. (Whether a piece rate or comparison with a fixed standard is preferred depends upon which is in some sense "closer" to the optimal general compensation scheme.)

\footnotetext{
${ }^{1}$ Nalebuff and Stiglitz (1981) compare tournaments and independent contracts using a somewhat different specification of the production technology. In their model the shock is multiplicative, so that it affects the marginal product of effort, making their results difficult to compare with ours. Holmström (1982) examines arbitrary relative performance schemes for risk-averse agents. He shows, among other things, that for either additive or multiplicative shocks, because the mean output level is a sufficient statistic for all of the information about the common shock, optimal contracts can be designed in which each agent's reward depends only on his own output level and on the mean output level. This is very close to our proposition 1.
} 
To see why a tournament performs well when the common shock is important and badly when it is unimportant, note that competing in a tournament is like being judged against a standard that is a random variable (the opponent's output). This is useful if the random standard is highly correlated with the random component of the agent's own output (the case when the randomness in the opponent's output is due largely to the common shock) and detrimental otherwise, because it introduces additional "noise" into the relationship between effort and compensation (the case when the randomness in the opponent's output is due largely to randomness in the output from effort).

Compensation schemes that base an agent's reward on the performance of his peers as well as his own take advantage of the fact that the vector of output levels for the whole group is a source of information about the common shock-which by assumption the principal cannot observe directly. How can the principal make use of this information? Optimal compensation schemes for groups of agents can, in general, have arbitrary and complicated functional forms depending on assumptions about tastes, technology, and distributions for the error terms. In practice, on the other hand, rather simple schemes are often used. Consequently, one is led to study the properties of simple schemes like tournaments-to understand when they perform "almost" as well as "optimal" multi-agent contracts. That is the viewpoint adopted here, where we extend the Lazear-Rosen model to compare the efficiency of tournaments and optimal independent contracts for any number of agents.

We consider a situation in which one risk-neutral principal employs a group of identical risk-averse agents. As in the Lazear-Rosen model, each agent's output is assumed to depend stochastically on his own effort and a common additive shock term. The common shock might represent economic conditions which affect all of the agents. We allow agents to observe private signals, correlated with this common shock, before they choose their effort levels. The realized output of each agent then is a stochastic function of his effort and the value of the common shock. The principal observes only the output levels of the agents.

We assume throughout that the principal is constrained to offer a fixed minimum level of expected utility to each agent, so that we can judge the relative performance of contracts and tournaments by examining the expected payoff of the principal. The principal's objective function is the sum of the outputs of all the agents minus the sum of the rewards paid to all of them.

We show that for any finite number of agents, in the absence of a common error term, using the optimal tournament is dominated by using optimal independent contracts. In the absence of a common 
shock, the output levels of the rest of the group convey no information about the effort level of an agent. Using a tournament in this case only introduces extraneous noise into the payoff function that agent faces. Because the agents are risk averse, this is costly for the principal.

Conversely, given any group of at least two agents, if the distribution of the common error term is sufficiently diffuse, then the optimal tournament dominates using optimal independent contracts. In this case using tournaments eliminates a major source of noise while adding a relatively minor one.

Finally, given any fixed distribution for the common error term, for a sufficiently large number of agents, using the optimal tournament dominates using optimal independent contracts. In fact, if the number of agents is sufficiently large, a principal who cannot observe the value of the common shock and uses an optimal tournament can do as well as a principal who can observe the value of the shock and uses general, interdependent contracts. For a large enough group of agents, an agent's rank order is an extremely accurate signal about his output level net of the common additive shock.

The rest of the paper is organized as follows. In Section II tastes, technology, distributions, and feasible sets of tournaments and contracts are described; in Section III tournaments and independent contracts are compared; and in Section IV the conclusions are discussed.

\section{The Model}

We consider the problem faced by a principal who employs a fixed group of agents, $i=1, \ldots, n$. The agents are all identical ex ante. The preferences of each agent $i$ over his income, $m_{i}$, and his effort, $x_{i}$, are represented by the von Neumann-Morgenstern utility function

$$
U^{i}\left(m_{i}, x_{i}\right)=u\left(m_{i}\right)-x_{i}, m_{i} \geqslant 0, x_{i} \geqslant 0, i=1, \ldots, n,
$$

where $u: R_{+} \rightarrow[0, B]$ is strictly increasing and strictly concave. ${ }^{2}$

\footnotetext{
${ }^{2}$ The fact that the utility function is additively separable in income and effort (that there are no income effects) is important. Without this assumption the analysis would be considerably more complicated, and it is not clear in what direction the results would change-it would almost surely depend on whether income raised or lowered the marginal disutility of effort. However, given the additively separable form, the fact that "effort" enters linearly into each agent's utility function involves no loss of generality. Because effort is never aggregated across agents, units of effort are simply defined as whatever causes units of disutility to the agent. Under this units convention $F$ reflects both the concavity of the agent's utility function in "hours" (or some other standard unit of measurement), as well as the concavity of the production function in hours. If at least one of these functions is strictly concave, then (3) holds. The agent's utility of income is bounded to avoid problems of the type discussed by Mirrlees (1975).
} 
The output of agent $i, y_{i}$, depends stochastically on his effort level, $x_{i}$. In particular,

$$
y_{i}=z_{i}+\eta
$$

where $\eta \in R$ is a random variable affecting all of the agents and $z_{i}$ is a random variable whose distribution depends on $x_{i}$. Let $F\left(; x_{i}\right)$ denote the conditional distribution function for $z_{i}$ given $x_{i}$; since the agents are identical ex ante, $F$ does not depend on $i$. Assume that for any effort level $x \geqslant 0$, the distribution function for output, $F(; x)$, has a continuous density function $f(; x)$ which is positive everywhere and continuously differentiable in $x$.

The agents observe private signals about $\eta$ before choosing their effort levels; let $\sigma_{i} \in R$ denote the signal observed by agent $i$ and let $G$ denote the distribution function for $(\eta, \sigma)$. Note that this formulation includes situations where all agents observe the same signal, independent signals, signals that reveal $\eta$ completely, and signals that are uncorrelated with $\eta$.

The value of $z_{i}$ is not known to the agent until after his choice of $x_{i}$ is made. We assume that $z_{i}$ and $(\eta, \sigma)$ are independent and that $\eta$ has zero mean:

$$
\int \eta d G(\eta, \sigma)=0 .
$$

(Except where otherwise indicated, integration is over the entire range.) This specification, though quite general, contains one special feature that is important to the subsequent development of the model. All agents are identical, except in their information about $\eta$. In particular, every agent, regardless of his assessment of $\eta$, still believes that his output and that of every other agent have the same mean if they take the same actions. Moreover, since the $z_{i}$ 's are independently and identically distributed for every common value of the $x_{i}$ 's, so will be the $y_{i}$ 's.

For example, the agents might be salesmen in a certain region whose individual territories are symmetrical and whose sales depend partly on regional conditions $(\eta)$, unobservable to the national office. The salesmen might all observe regional conditions perfectly $\left(\sigma_{i} \equiv \eta\right.$, all $i$ ), or might observe the same imperfect signal $\left(\sigma_{i} \equiv \sigma_{j} \neq \eta\right.$, all $\left.i, j\right)$, or might observe different imperfect signals $\left(\sigma_{i} \neq \sigma_{j} \neq \eta\right.$, all $\left.i, j\right)$.

The principal's problem is to design a reward structure for the $n$ agents. The principal is risk neutral and seeks to maximize the expected sum of the outputs net of total payments to the agents:

$$
E\left[\sum_{i=1}^{n}\left(y_{i}-m_{i}\right)\right]
$$

By assumption the principal observes only the output levels of the 
agents, $y=\left(y_{1}, y_{2}, \ldots, y_{n}\right)$; he cannot directly observe either the agents' effort levels or the realization of any random variable. Under independent contracts agent $i$ 's reward depends only on his own output level, $y_{i}$, while under a tournament it depends only on the rank order of $y_{i}$ in $y$.

Given any reward structure, the problem facing each agent is to choose a level of effort. First consider the situation under independent contracts. Since the agents are identical, we can consider the problem facing a representative agent $i$. It is convenient to think of the principal as constructing the reward function in terms of utility. For any reward function $R(y)$, let $v(y)$ be defined as its representation in utility terms, $v(y) \equiv u[R(y)]$. The cost to the principal of providing this level of utility is then given by $\gamma[v(y)]$, where $\gamma \equiv u^{-1}$. Since $u$ is strictly increasing and strictly concave, $\gamma$ is strictly increasing and strictly convex. Agent $i$ observes $\sigma_{i}$ and then chooses the level of effort that maximizes his expected utility. Because the optimal level of effort will depend on the value of $\sigma_{i}$, the optimal decision rule for the agent is a function $X\left(\sigma_{i}\right)$.

The principal's problem is to choose $(v, X)$ to maximize (4) subject to the two constraints that $X$ be an optimal decision rule for the agent given $v$ and that the expected utility of the agent be at least $u^{0}$.

The decision rule of each agent depends upon the joint information system of the agents as summarized in the distribution $G$, upon the decision rules chosen by all other agents, and upon the reward function. Throughout this paper we concentrate on symmetric equilibria, that is, on solutions to the principal's problem in which all agents choose the same decision rule ex ante, their behavior ex post differing only as a result of the different signals ("news") they receive.

Thus, given $G$, define $S_{c i}(G)$ to be the set of contracts that are feasible for the $i$ th agent:

$$
\begin{aligned}
S_{c i}(G) & \equiv\left\{(v, X) \mid v: R_{+} \rightarrow[0, B], X: R \rightarrow R_{+} ;\right. \\
& X\left(\sigma_{i}\right) \in \underset{x}{\operatorname{argmax}} \int v(y) \int f(y-\eta ; x) d G\left(\eta, \sigma_{-i} \mid \sigma_{i}\right) d y-x, \forall \sigma_{i} \\
& \left.\iint\left[v(y)-X\left(\sigma_{i}\right)\right] f\left[y-\eta ; X\left(\sigma_{i}\right)\right] d G(\eta, \sigma) d y \geqslant u^{0}\right\} .
\end{aligned}
$$

Here, $X(\cdot)$ is the common decision rule. Equation (5a) expresses its optimality for each signal, $\sigma_{i}$, the integration being taken with respect to $\eta$ and $y$, or equivalently $\eta$ and $\left(z_{i}\right), i=1, \ldots, n$, which are random variables at the time $x_{i}$ is chosen. Equation (5b) represents the constraint on each agent's utility. As we consider symmetric equilibria and as $u^{0}$ is the same for each agent, this constraint takes the form of a single inequality.

Define $P_{c i}(v, X, G)$ to be the expected payoff of the principal from the contract $(v, X)$ : 


$$
P_{c i}(v, X, G) \equiv \iint\{y-\gamma[v(y)]\} f\left[y-\eta ; X\left(\sigma_{i}\right)\right] d y d G(\eta, \sigma) .
$$

The feasible set is always nonempty, since it always contains the "noincentive" contract: $\left(v^{0} \equiv u^{0}, X^{0} \equiv 0\right) \in S_{c i}(G)$, for all $G$, $i$. Note that the expected payoff to the principal under this contract, call it $P^{0}$, is independent of $G$ :

$$
\begin{aligned}
P^{0} \equiv P_{c i}\left(v^{0}, X^{0}, G\right) & =\iint\left[z+\eta-\gamma\left(u^{0}\right)\right] f(z ; 0) d z d G(\eta, \sigma) \\
& =\int z f(z ; 0) d z-\gamma\left(u^{0}\right) .
\end{aligned}
$$

Next consider the situation under a tournament. As above, it is convenient to express rewards in terms of utility. In an $n$-person tournament with prizes $\left(W_{1}, W_{2}, \ldots, W_{n}\right)$, define $w=\left(w_{1}, w_{2}, \ldots, w_{n}\right)$ by $w_{i} \equiv u\left(W_{i}\right), \forall i$. (We use the numbering conventional in the study of order statistics: "first place" is the lowest outcome and $w_{1}$ is the prize received by the agent with the lowest output, etc.) We will consider symmetric Nash equilibria of the game in which each agent's strategy is his effort level. ${ }^{3}$

Since each agent's output is given by $y_{i}=z_{i}+\eta, y_{i} \geqslant y_{j} \Leftrightarrow z_{i} \geqslant z_{j}$. That is, the rank order of the outputs depends only on the $z_{i}$ 's and not on $\eta$. Therefore, the realization of $(\eta, \sigma)$ does not affect the game played by the agents and the equilibrium effort level will be indepen-

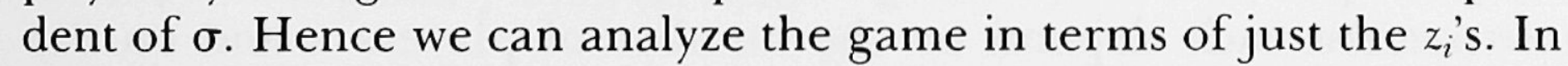
an $n$-person tournament, agent $i$ wins prize $w_{j}$ if and only if $z_{i}$ is the $j$ th-order statistic of $\left(z_{1}, \ldots, z_{n}\right)$. Define

$$
\phi_{j n}(z ; x) \equiv \frac{n !}{(n-j) !(j-1) !} f(z ; x) F^{j-1}(z ; x)[1-F(z ; x)]^{n-j} .
$$

That is, $\phi_{j n}(z ; x)$ is the density function for the $j$ th-order statistic in a sample of size $n$ drawn from the distribution $F(; x)$.

As above, the principal is constrained to offer the agents an expected utility of at least $u^{0}$. We are interested only in tournaments that have symmetric Nash equilibria. Given $n$ and $G$, the set of feasible $n$ person tournaments, $\hat{S}_{T}(n, G)$, is defined by

$$
\begin{gathered}
\hat{S}_{T}(n, G) \equiv\left\{(w, \bar{x}) \mid w \in[0, B]^{n}, \bar{x} \in R_{+} ;\right. \\
\bar{x} \in \underset{x}{\operatorname{argmax}} \frac{1}{n} \sum_{j=1}^{n} w_{j} \int \frac{f(z ; x)}{f(z ; \bar{x})} \phi_{j n}(z ; \bar{x}) d z-x ; \\
\left.\frac{1}{n} \sum_{j=1}^{n} w_{j}-\bar{x} \geqslant u^{0}\right\},
\end{gathered}
$$

${ }^{3}$ For arbitrary prize structures, there may be no Nash equilibrium, symmetric or otherwise. This is of no importance to us, since we are considering only tournaments that are designed so that they do have a symmetric Nash equilibrium. The restriction to tournaments with symmetric equilibria is purely on the grounds of tractability. 
The integrand in (6a) is the probability that the agent doing the maximization, and choosing $x$ while all other agents choose $\bar{x}$, is in the $j$ th place at the measured output level $y=z+\eta$. This can be seen by direct comparison with the definition of $\phi_{j n}$ above. Thus, the value of the integral is simply the probability of this agent being $j$ th in the sample given the choices of $x$ and $\bar{x}$.

Note that $\hat{S}_{T}(n, G)$ is independent of $G$ for all $G$ satisfying (3). Hence, for simplicity we write $\hat{S}_{T}(n, G) \equiv S_{T}(n)$ from this point on.

Given $n, G$, and $(w, \bar{x}) \in S_{T}(n)$, let $\hat{P}_{T}(n, w, \bar{x}, G)$ denote the principal's expected net payoff per agent under the tournament $(w, \bar{x})$ :

$$
\begin{aligned}
\hat{P}_{T}(n, w, \bar{x}, G) & \equiv \iint y f(y-\eta ; \bar{x}) d G(\eta, \sigma) d y-\frac{1}{n} \sum_{j=1}^{n} \gamma\left(w_{j}\right) \\
& =\int z f(z ; \bar{x}) d z-\frac{1}{n} \sum_{j=1}^{n} \gamma\left(w_{j}\right),
\end{aligned}
$$

where the second line makes use of (2) and (3). Since $G$ does not appear in the second line, for simplicity we write $\hat{P}_{T}(n, w, \bar{x}, G)=$ $P_{T}(n, w, \bar{x})$. We summarize these results in lemma 1 .

Lemma 1: The set of feasible tournaments, the expected payoff of the principal under any feasible tournament, and hence the optimal tournament each depend on the number of players $n$ and on the distribution function $F$ but not on the distribution function $G$.

Lemma 1 is interesting in its own right, for it says that tournaments are robust against lack of information or lack of agreement about $G$. It will also be useful for our later results. Note that the set of feasible tournaments is always nonempty, since it always contains the "noincentive" tournament, $\left[\left(u^{0}, u^{0}, \ldots, u^{0}\right), 0\right] \in S_{T}(n)$, for all $n$. The payoff per agent to the principal under this tournament is $P^{0}$.

\section{Comparison of Tournaments and Contracts}

First we will show that for any number of agents $n$ and any function $F$ satisfying (2), if there is no common error term, that is, if

$$
\int_{\sigma \in R^{n}} d G(\eta, \sigma) \equiv \begin{cases}0 & \text { for } \eta<0 \\ 1 & \text { for } \eta \geqslant 0\end{cases}
$$

then for any feasible tournament there exists a feasible contract that dominates it. ${ }^{4}$ Note that if $G$ satisfies (7), then it also satisfies (3).

Proposition 1: For any $F, G$ satisfying $(7)$, and $n \geqslant 2$, given $(w, \bar{x})$

${ }^{4}$ This result is (almost) a special case of theorem 7 in Holmström (1982). (In Holmström's model agents do not have any information about the common shock.) Proposition 1 is included here for the sake of completeness. 
$\in S_{T}(n)$, there exists $(v, X) \in S_{c i}(G), i=1, \ldots, n$, such that

$$
P_{c i}(v, X, G) \geqslant P_{T}(n, w, \bar{x}), \quad i=1, \ldots, n .
$$

The inequality is strict unless $(w, \bar{x})=\left[\left(u^{0}, u^{0}, \ldots, u^{0}\right), 0\right]$.

Proof: Let $F, G, n \geqslant 2$, and $(w, \bar{x}) \in S_{T}(n)$ be given. We will show that the contract $(v, X)$ defined by

$$
\begin{array}{rlrl}
v(y) & \equiv \frac{1}{n} \sum_{j=1}^{n} w_{j} \phi_{j n}(y ; \bar{x}) / f(y ; \bar{x}), & & \text { for all } y ; \\
X\left(\sigma_{i}\right)=\bar{x}, & \text { for all } \sigma_{i},
\end{array}
$$

satisfies the required conditions.

The contract above has the following interpretation. The utility assigned to output $y$ is the expected value of the $w_{j}$, where the probability assigned to $w_{j}$ is the probability that $y$ will be the $j$ th-order statistic, given that everyone takes the action $\bar{x}$. It is automatically monotonic in $y$ if the $w_{j}$ are increasing in $j$.

First we will show that the proposed contract satisfies (5a) and (5b). Since $G$ satisfies (7), any agent's optimal effort function under $v$ is given by

$$
\begin{aligned}
X\left(\sigma_{i}\right) & =\underset{x}{\operatorname{argmax}} \int v(z) f(z ; x) d z-x \\
& =\underset{x}{\operatorname{argmax}} \int \frac{1}{n} \sum_{j=1}^{n} w_{j} \frac{\phi_{j n}(z ; \bar{x})}{f(z ; \bar{x})} f(z ; x) d z-x, \text { for all } \sigma_{i} .
\end{aligned}
$$

Since $(w, \bar{x})$ satisfies $(6 \mathrm{a}), X\left(\sigma_{i}\right) \equiv \bar{x}$ and $(5 \mathrm{a})$ is satisfied.

Moreover, since $(w, \bar{x})$ satisfies ( $6 \mathrm{~b})$, the expected utility of the agent under $v$ is given by

$$
\int v(z) f(z ; \bar{x}) d z-\bar{x}=\int \frac{1}{n} \sum_{j=1}^{n} \frac{\phi_{j n}(z ; \bar{x})}{f(z, \bar{x})} w_{j} f(z ; \bar{x}) d z-\bar{x} \geqslant u^{0} .
$$

Hence (5b) is also satisfied.

The expected utility of the principal is higher under $(v, X)$ than under $(w, \bar{x})$, since

$$
\begin{aligned}
P_{c}(v, X, G) & =\int\{z-\gamma[v(z)]\} f(z ; \bar{x}) d z \\
& =\int z f(z ; \bar{x}) d z-\int \gamma\left[\frac{1}{n} \sum_{j=1}^{n} \frac{\phi_{j n}(z, \bar{x})}{f(z ; \bar{x})} w_{j}\right] f(z ; \bar{x}) d z \\
& \geqslant \int z f(z, \bar{x}) d z-\frac{1}{n} \sum_{j=1}^{n} \gamma\left(w_{j}\right) \int \phi_{j n}(z, \bar{x}) d z \\
& =P_{T}(n, w, \bar{x}) .
\end{aligned}
$$

The inequality follows from Jensen's inequality and the fact that $\gamma$ is 
strictly convex. If $w \neq(\bar{w}, \ldots, \bar{w})$, the inequality is strict. If $w=(\bar{w}$, $\ldots, \bar{w})$, then $\bar{x}=0$, and unless $\bar{w}=u^{0}$, the contract $\left(v \equiv u^{0}, X \equiv 0\right)$ dominates $(w, \bar{x})$. Q.E.D.

The contract $v(y)$ above assigns to each $y$ the mean payoff that the player with realized output $y$ would get in a tournament in which all agents have chosen $\bar{x}$. Clearly, the random tournament payoff is riskier than the random contract payoff, as it is the latter composed with the additional risk in rank order-risk which depends only upon other agents' outcomes.

An obvious corollary of proposition 1 is that when there is no common error term, the optimal contract dominates the optimal tournament.

Corollary 1: Let $F, G$ satisfying ( 7 ), and $n \geqslant 2$ be given. Then

$$
\max _{(v, X) \in S_{a}(G)} P_{c i}(v, X, G) \geqslant \max _{(w, \bar{x}) \in S_{T}(n)} P_{T}(n, w, \bar{x}) \text {, for all } i .
$$

The inequality is strict unless $\left(v \equiv u^{0}, X \equiv 0\right)$ maximizes the left-hand side.

Next, we will compare independent contracts and tournaments as the common error term becomes diffuse. We will consider sequences $\left\{G_{k}\right\}_{k=1}^{\infty}$ such that, for all $k$,

$G_{k}$ satisfies (2);

$G_{k}$ has a density function $g_{k}$;

$$
\int g_{k}\left(\eta, \sigma_{-i} \mid \sigma_{i}\right) d \sigma_{i} \equiv g_{k i}\left(\eta \mid \sigma_{i}\right)<1 / k, \text { for all } \eta, \sigma_{i}, i \text {. }
$$

The third condition in (8) says that as $k \rightarrow \infty$, the densities $g_{k i}$ become very small, meaning that the distributions spread out very thinly.

In proposition 2 we show that for any sequence $\left\{G_{k}\right\}$ satisfying (8), for all $k$ sufficiently large, the optimal contract is the "no-incentive" contract $\left(v \equiv u^{0}, X \equiv 0\right)$. Hence the principal's expected payoff under the optimal contract falls to $P^{0}$ along the sequence. However, as shown above, the optimal tournament and the principal's expected payoff under it - which is at least $P^{0}$-will be unchanged along the sequence.

Proposition 2: Let $F,\left\{G_{k}\right\}_{k=1}^{\infty}$ satisfying (8), and $n \geqslant 2$ be given. Assume that $F_{x}(z ; x)$ is a function of bounded variation in $z$, for all $x \geqslant$ 0 , and that the bound, $M$, is uniform in $x$. Then there exists $K$ such that, for all $k>K$,

$$
\max _{(w, \bar{x}) \in S_{T}(n)} P_{T}(w, \bar{x}, n) \geqslant \max _{(v, X) \in S_{c}\left(G_{k}\right)} P_{c i}\left(v, X, G_{k}\right), \quad i=1, \ldots, n .
$$

The inequality is strict unless the left-hand side is equal to $P^{0}$.

Proof: Let $\left\{\left(v_{k i}^{*}, X_{k i}^{*}\right)\right\}$ be a sequence of optimal contracts for agent $i$. 
Note that

$$
X_{k i}^{*}\left(\sigma_{i}\right)>0 \Rightarrow 1=\int v_{k i}^{*}(y) \int f_{x}\left[y-\eta ; X_{k i}^{*}\left(\sigma_{i}\right)\right] g_{k i}\left(\eta \mid \sigma_{i}\right) d \eta d y .
$$

However, since $f_{x}$ is of bounded variation, (8) implies that

$$
\begin{aligned}
\lim _{k \rightarrow \infty}\left|\int f_{x}\left(y-\eta ; X_{k i}^{*}\right) g_{k i}\left(\eta \mid \sigma_{i}\right) d \eta\right| & \leqslant \lim _{k \rightarrow \infty} \frac{1}{k} \int\left|f_{x}\left(y-\eta ; X_{k i}^{*}\right)\right| d \eta \\
& \leqslant \lim _{k \rightarrow \infty} \frac{M}{k}=0 .
\end{aligned}
$$

Since $v_{k i}^{*}(y) \in[0, B]$, for all $y,(10)$ cannot hold. Hence for $k$ sufficiently large $\left(v_{k i}^{*} \equiv u^{0}, X_{k i}^{*} \equiv 0\right)$, and $P_{c}\left(v_{k i}^{*}, X_{k i}^{*}, G_{k}\right)=P^{0}$.

By lemma 1, $S_{T}$ and $P_{T}$ are independent of $G$, so that the left-hand side of $(9)$ is at least $P^{0}$ and is constant along the sequence $\left\{G_{k}\right\}$.Q.E.D.

Our final result concerns the relative efficiency of tournaments and contracts as the number of agents grows. We will consider sequences of distributions $\left\{G_{n}\left(\eta, \sigma_{1}, \ldots, \sigma_{n}\right)\right\}_{n=2}^{\infty}$ such that the marginal distribution function for $\eta$ is unchanged throughout:

$$
\int_{\sigma \in R^{n}} d G_{n}(\eta, \sigma)=\bar{G}(\eta), \text { for all } n=2,3, \ldots
$$

We will show that as $n \rightarrow \infty$, not only does the optimal tournament dominate the optimal contract, but in fact the optimal tournament approaches the full-information solution. That is, as $n \rightarrow \infty$ the principal does as well as if he could observe $\eta$ directly. There are two steps in the proof.

In lemma 2 we show that any contract for which the payoff function is piecewise continuous and the agent's optimal effort level is unique can be approximated arbitrarily closely by a payoff function that is a step function and that changes the effort level chosen by agents in a continuous way. Thus, if there are enough steps, the payoff of the principal is approximately unchanged. Then in lemma 3 we show that each of these step-function contracts can be approximated arbitrarily closely (in the sense that efforts and payoffs are close) by a tournament with a sufficiently large number of players. Hence the principal's expected payoff is, again, approximately unchanged.

Now suppose that $G$ is given by $(7)(\eta=0$ with probability 1$)$, so that the principal knows $\eta$ ex ante, and suppose that he designs the optimal (full-information) contract. These approximation arguments show that a tournament can yield almost as large an expected payoff when $n$ is large. Moreover, the tournament's efficiency is unaffected by changes in $G$ (the distribution of $\eta$ and the agent's information about $\eta$ ), so that the same tournament's payoff remains arbitrarily 
close to the full-information payoff for any $G$. In contrast, under the optimal contract, if the principal in fact does not observe $\eta$, his expected payoff will, in general, fall short.

Let $G$ satisfying (7) be given. As noted above, when $G$ satisfies (7) we can restrict attention to contracts $(v, X)$ for which $X\left(\sigma_{i}\right) \equiv \bar{X}$ is a constant function. For these contracts $S_{c i}=S_{c}$ and $P_{c i}=P_{c}$, for all $i$. First we will show that given any feasible contract $(v, X)$ we can construct a sequence of contracts $\left\{\left(v_{k}, \bar{X}_{k}\right)\right\}_{k=1}^{\infty}$ such that $v_{k}$ is a step function with $k$ steps, $\bar{X}_{k}$ is a constant function, and $v_{k} \rightarrow v$ in measure.

Let $[v(y), \bar{X}] \in S_{c}(G)$ be given, and let $I_{k 1}, \ldots, I_{k k}$ be intervals corresponding to quantiles of the distribution $F(; \bar{X})$ :

$$
\begin{aligned}
I_{k j} & \equiv\{z \mid(j-1) / k<F(z ; \bar{X}) \leqslant j / k\}, \\
j & =1, \ldots, k ; k=1,2,3, \ldots .
\end{aligned}
$$

Let $\bar{v}_{k 1}, \ldots, \bar{v}_{k k}$ be the expected payoff of the agent under $(v, \bar{X})$ on each of these intervals:

$$
\bar{v}_{k j} \equiv \int_{I_{k j}} v(z) f(z ; \bar{X}) d z, \quad j=1, \ldots, k ; k=1,2, \ldots
$$

Next, define the step function $\hat{v}_{k}(z)$ by

$$
z \in I_{k j} \Rightarrow \hat{v}_{k}(z)=\bar{v}_{k j}, \quad \text { for all } z, k,
$$

as shown in figure 1 . Note that as $k \rightarrow \infty, \hat{v}_{k}(z) \rightarrow v(z)$ in measure. Finally, define

$$
\begin{gathered}
\bar{X}_{k} \equiv \underset{x}{\operatorname{argmax}} \int \hat{v}_{k}(z) f(z ; x) d z-x, \text { for all } k ; \\
c_{k} \equiv \bar{u}+\bar{X}_{k}-\int \hat{v}_{k}(z) f\left(z ; \bar{X}_{k}\right) d z, \text { for all } k ; \\
v_{k}(z)=\hat{v}_{k}(z)+c_{k}, \quad \text { for all } z, k,
\end{gathered}
$$

where $\bar{u}$ is the agent's expected utility under $(v, \bar{X})$. Note that by construction, for $G$ satisfying (7), $\left(v_{k}, \bar{X}_{k}\right) \in S_{c}(G)$, for all $k$.

Lemma 2: Let $F, G$ satisfying (7), and $(v, \bar{X}) \in S_{c}(G)$ be given. If $v$ is piecewise continuous and if $\bar{X}$ is the unique solution of

$$
\max _{x} \int v(z) f(z ; x) d z-x
$$

then for the sequence $\left\{\left(v_{k}, \bar{X}_{k}\right)\right\}_{k=1}^{\infty}$ defined by (12)-(17),

$$
\lim _{k \rightarrow \infty} P_{c}\left(v_{k}, \bar{X}_{k}, G\right)=P_{c}(v, \bar{X}, G),
$$

and for $k$ sufficiently large, $\bar{X}_{k}$ is the unique solution of

$$
\max _{x} \iint v_{k}(y) f(y-\eta ; x) d G(\eta) d y-x .
$$




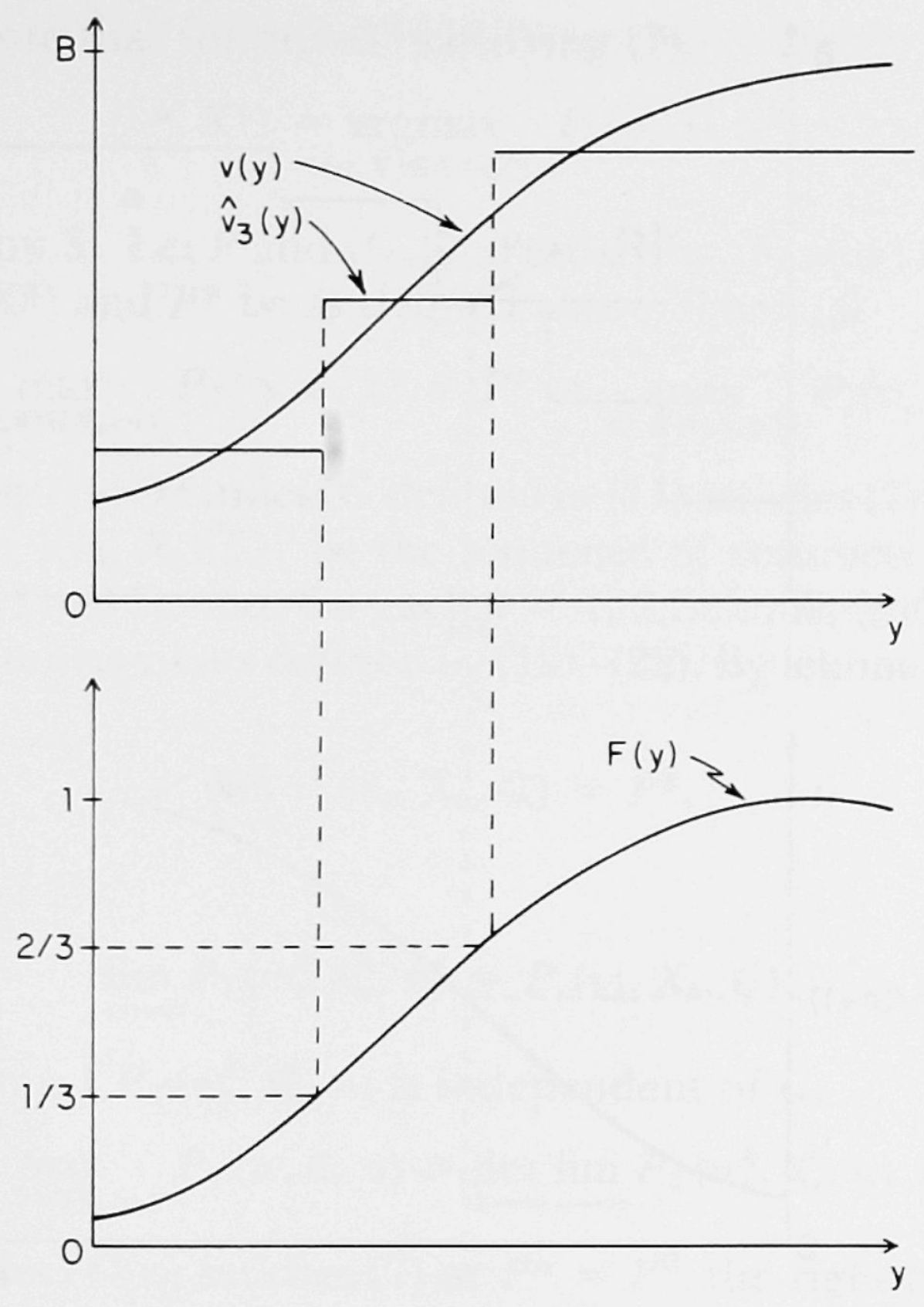

FIG. 1

Proof: See the Appendix.

Next, we will show that for $G$ satisfying $(7)$, given any contract $(v, \bar{X})$ $\in S_{c}(G)$, where $v$ is a step function and $\bar{X}$ is a constant function, we can construct a sequence of tournaments that approximate it. Define $y_{n i}$ by $F\left(y_{n i} ; \bar{X}\right) \equiv i /(n+1)$, as shown in figure 2 , and define $\hat{w}_{n i}$ by

$$
\hat{w}_{n i} \equiv v\left(y_{n i}\right) \text {. }
$$

Then where it exists define

$$
\bar{x}_{n}=\underset{x}{\operatorname{argmax}} \int f(z ; x) \frac{1}{n} \sum_{i=1}^{n} \hat{w}_{n i} \frac{\phi_{\text {in }}\left(z ; \bar{x}_{n}\right)}{f\left(z ; \bar{x}_{n}\right)} d z-x,
$$

and let

$$
\begin{gathered}
a_{n} \equiv \bar{u}+\bar{X}_{n}-\frac{1}{n} \sum_{i=1}^{n} \hat{w}_{n i}, \\
w_{n i} \equiv \hat{w}_{n i}+a_{n}, \quad i=1, \ldots, n .
\end{gathered}
$$




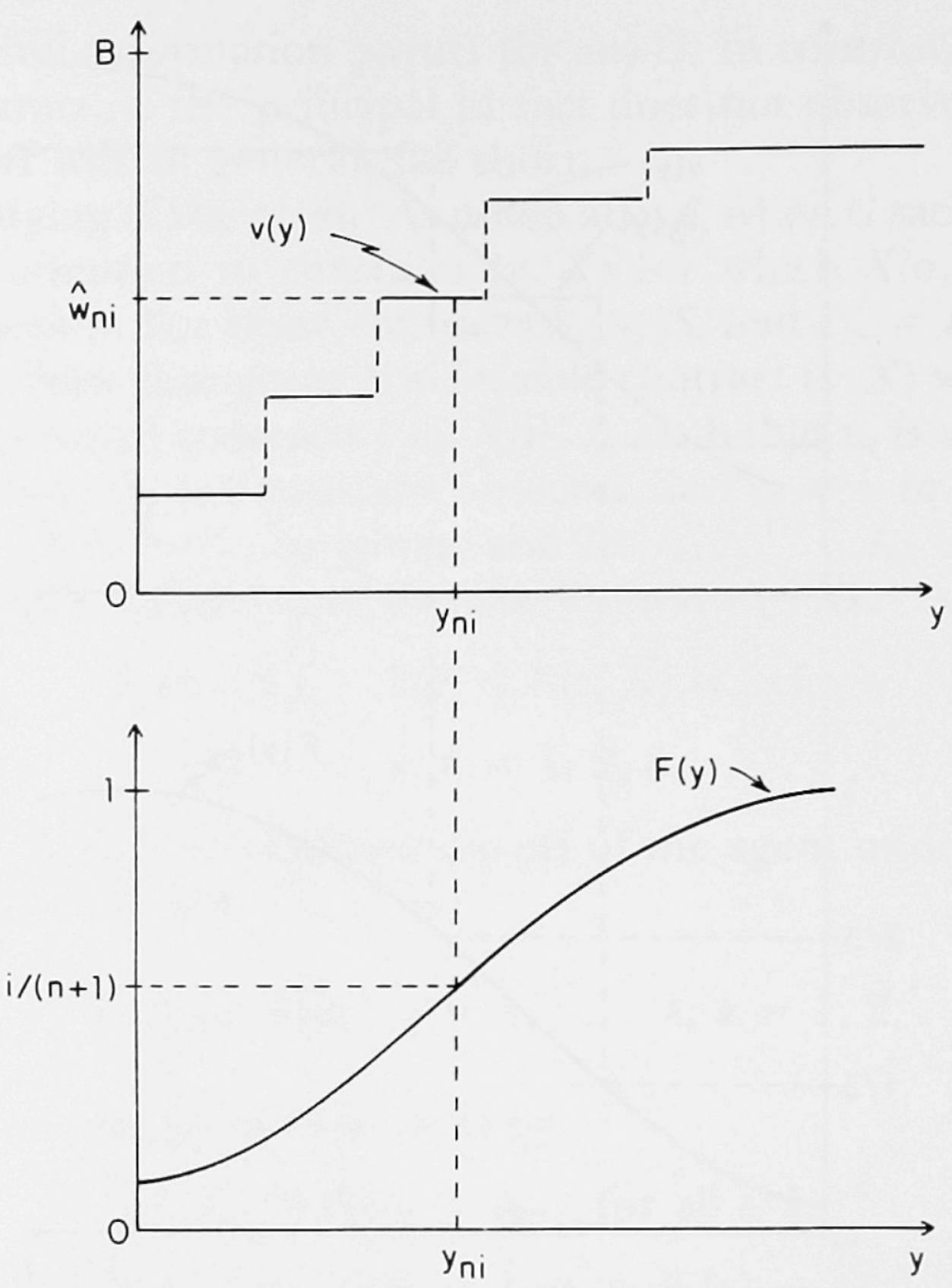

FIG. 2

Lemma 3: Let $F, G$ satisfying (7), and $(v, \bar{X}) \in S_{c}(G)$ be given, where $v$ is a step function, $\bar{X}$ is a constant function, and $\bar{X}$ is the unique solution of (18). Let $\left\{\left(\hat{w}_{n}\right)\right\}_{n=1}^{\infty}$ and $\left\{\left(w_{n}, \bar{x}_{n}\right)\right\}_{n=1}^{\infty}$ be the sequences defined by (19)-(22). For $n$ sufficiently large, $\bar{x}_{n}$ as defined by (20) exists and $\left(w_{n}, \bar{x}_{n}\right) \in S_{T}(n)$. Moreover

$$
\lim _{n \rightarrow \infty} P_{T}\left(n, w_{n}, \bar{x}_{n}\right)=P_{c}(v, \bar{X}, G) .
$$

Proof: See the Appendix.

As a reference point, we want to define the optimal contract and expected payoff for a principal who can observe $\eta$. If the principal can observe $\eta$ directly, the optimal contract $\left(v^{*}, X^{*}\right)$ is independent of $G$ and has the form

$$
\begin{aligned}
v(y, \eta) & =v^{*}(y-\eta), & & \text { for all } y, \eta \\
X(\eta) & =X^{*}, & & \text { for all } \eta .
\end{aligned}
$$

The principal's expected payoff in this case is given by

$$
P^{*} \equiv \int\left[z-v^{*}(z)\right] f\left(z ; X^{*}\right) d z .
$$


Moreover, note that for any $G^{0}$ satisfying (7),

$$
\left(v^{*}, X^{*}\right) \underset{(v, X) \in S_{c}\left(G^{0}\right)}{\operatorname{argmax}} P_{c}\left(v, X, G^{0}\right) .
$$

Proposition 3: Let $F$ and $\left\{G_{n}\right\}_{n=2}^{\infty}$ satisfying (3) and (11) be given, and let $\left(v^{*}, X^{*}\right)$ and $P^{*}$ be as defined above. Then,

$$
\lim _{n \rightarrow \infty} \max _{(w, \bar{x}) \in S_{T}(n)} P_{T}(n, w, \bar{x})=P^{*} \geqslant \max _{(v, X) \in S_{c}(G)} P_{c}(v, X, G) .
$$

The inequality is strict unless $\bar{G}$ defined in (11) satisfies (7) or $P^{*}=P^{0}$.

Proof: Let $\left\{\left(v_{k}, X_{k}\right)\right\}_{k=1}^{\infty}$ be the sequence of contracts defined for $\left(v^{*}, X^{*}\right)$ by $(12)-(17)$, and, for each $k=1,2, \ldots$, let $\left\{\left(w_{n}^{k}, \bar{x}_{n}^{k}\right)\right\}$ be the sequence of tournaments defined by (19)-(22). By lemma 2 , for any $G$ satisfying (7),

$$
\lim _{k \rightarrow \infty} P_{c}\left(v_{k}, X_{k}, G\right)=P^{*},
$$

and by lemma 3 ,

$$
\lim _{n \rightarrow \infty} P_{T}\left(w_{n}^{k}, \bar{x}_{n}^{k}, n\right)=P_{c}\left(v_{k}, X_{k}, G\right) .
$$

Since by lemma $1 P_{T}\left(w_{n}^{k}, \bar{x}_{n}^{k}, n\right)$ is independent of $G$,

$$
\lim _{n \rightarrow \infty} \max _{(w, \bar{x}) \in S_{T}(n)} P_{T}(w, \bar{x}, n) \geqslant \lim _{k \rightarrow \infty} \lim _{n \rightarrow \infty} P_{T}\left(w_{n}^{k}, \bar{x}_{n}^{k}, n\right)=P^{*} .
$$

However, unless $\bar{G}(\eta)$ satisfies (7) or $P^{*}=P^{0}$, the right-hand side of (24) is strictly less than $P^{*}$ (Holmström 1979, corollary 2). Q.E.D.

\section{Conclusions}

In the model above, all agents' output levels are subject to the same random shock. Thus, the output levels of the group provide the principal with information about the value of the common shock and consequently about the portion of any particular agent's output that is attributable to effort. Relative performance schemes-of which tournaments are an extreme form-allow the principal to make use of this information.

Obviously tournaments employ available information in a rather inefficient and inflexible way. In the model above, tournaments tend to reduce the randomness of any agent's compensation by filtering out the common shock term. However, they also tend to increase the randomness in any agent's compensation by making his reward depend on the idiosyncratic shocks of his peers. Propositions 1 and 2 show that the relative advantage of tournaments vis-à-vis contracts depends on which effect dominates.

Despite the fact that a tournament makes inefficient use of information, proposition 3 shows that this entails no loss if the number of 
agents is sufficiently large. In large groups, the rank order of an agent's observed output is a very accurate estimator of his output net of the common shock.

Tournaments are not, in general, "optimal" contracts. Why then are rankings so commonly used as an evaluation criterion? First, it may be substantially easier to determine agents' rankings than to measure their output levels. In addition, as shown in lemma 1, neither the set of feasible tournaments nor the optimal tournament depends on the distribution function for the common shock and agents' signals. This is an obvious advantage if that distribution is unknown or imprecisely known-as it would be, for example, in nonstationary environments. Because in a large group the inefficiency due to information loss is negligible, tournaments will perform very well in such settings.

\section{Appendix}

Proof of Lemma 2: Since $f(; x)$ is continuous in $x, \hat{v}_{k} \rightarrow v$ in measure, and $\bar{X}$ is the unique maximizer of (18), from (15) it follows that $\bar{X}_{k} \rightarrow \bar{X}$. Hence $c_{k} \rightarrow 0$ and $v_{k} \rightarrow \hat{v}_{k}$. By construction, then,

$$
\lim _{k \rightarrow \infty} \int \gamma\left[v_{k}(z)\right] f\left(z ; \bar{X}_{k}\right) d z=\int \gamma[v(z)] f(z ; \bar{X}) d z,
$$

so that the desired conclusion follows immediately.

Proof of Lemma 3: Consider a representative player $j$ in an $n$-person tournament with prizes $\hat{w}_{n}$. Suppose that the $n-1$ other players all adopt the effort level $\bar{X}$, and consider the conditional distribution of prizes for player $j$, given that his observed output is $y_{j}$. Unless $y_{j}$ is a point of discontinuity in the step function $v$, as the number of players increases, by the law of large numbers the conditional probability that $j$ has a rank order such that his prize is equal to $v\left(y_{j}\right)$ approaches one. That is,

$$
\lim _{n \rightarrow \infty} \frac{1}{n} \sum_{i=1}^{n} \hat{w}_{n i} \phi_{i n}(z ; \bar{X}) / f(z ; \bar{X})=v(z ; \bar{X}), \text { for almost all } z .
$$

Since $\bar{X}$ is the unique solution of (18), for $n$ sufficiently large, $\bar{x}_{n}$ as defined by (20) exists, and $\lim _{n \rightarrow \infty} \bar{x}_{n}=\bar{X}$. Thus (23) follows immediately.

\section{References}

Holmström, Bengt. "Moral Hazard and Observability." Bell J. Econ. 10 (Spring 1979): 74-91.

(1. "Moral Hazard in Teams," Bell J. Econ. 13 (Autumn 1982): 324-40.

Lazear, Edward P., and Rosen, Sherwin. "Rank-Order Tournaments as Optimum Labor Contracts." J.P.E. 89 (October 1981): 841-64.

Mirrlees, James. "The Theory of Moral Hazard and Unobservable Behavior, Part I." Mimeographed. Cambridge: Cambridge Univ., 1975.

Nalebuff, Barry, and Stiglitz, Joseph E. "Prizes and Incentives: Towards a General Theory of Compensation and Competition." Mimeographed. Princeton, N.J.: Princeton Univ., August 1981. 
Copyright of Journal of Political Economy is the property of University of Chicago Press and its content may not be copied or emailed to multiple sites or posted to a listserv without the copyright holder's express written permission. However, users may print, download, or email articles for individual use. 